Table des matières du tome XCVIII, fascicule 3

Pages

A. Lelek, The span and the width of continua . . . . . . . . . . . . . . . 181-199 A. Prószyński, On orthogonal decomposition of homogeneous polynomials . . . . 201-217 - Some functors related to polynomial theory . . . . . . . . . . . . . . . . 219-229 E. D. Tymchatyn, On finitely Suslinian continua . . . . . . . . . . . . . 231-247 R. Ger, On some functional equations with a restricted domain, in . . . . . . . . 249-272 H. Länger, $C$-S-maximal superassociative systems . . . . . . . . . . . . 273-277 W. G. Fleissner, Separation properties in Mare spaces . . . . . . . . . . . 279-286

Les FUNDAMENTA MATHEMATTEAE publient, en langues des congrès internationaux, des travaux consacrés à la Théorie des Ensembles, Topologie, Fondements de Mathématiques, Fonctions Réelles, Théorie Descriptive des Ensembles, Algèbre Abstraite

Chaque volume paraît en 3 fascicules

Adresse de la Rédaction:

FUNDAMENTA MATHEMATICAE, Sniadeckich 8, 00-950 Warszawa (Pologne) Adresse de l'Échange:

INSTITUT MATHÉMATIQUE, ACADÉMIE POLONAISE DES SCIENCES Sniadeckich 8, 00-950 Warszawa (Pologne)

Tous les volumes sont à obtenir par l'intermédiaire de

ARS POLONA, Krakowskie Przedmieście 7, 00-068 Warszawa (Pologne)

Correspondence concerning editorial work and manuscripts should be addressed to: FUNDAMENTA MATHEMATICAE, Śniadeckich 8, 00-950 Warszawa (Poland)

Correspondence concerning exchange should be addressed to:

INSTITUTE OF MATHEMATICS, POLISH ACADEMY OF SCIENCES, Exchange Sniadeckich 8, 00-950 Warszawa (Poland)

The Fundamenta Mathematicae are available at your bookseller or at ARS POLONA, Krakowskie Przedmieście 7, 00-068 Warszawa (Poland)

(C) Copyright by Państwowe Wydawnictwo Naukowe, Warszawa, 1978

DRUKARNIA UNIWERSYTETU JAGIELIONSKIEGOW KRAKOWI

\section{The span and the width of continua}

\author{
A. Lelek (Detroit, Mich.)
}

Abstract. The concept of the span is compared with Burgess' notion of the width. Some basic properties of them are established. The results are applied to an investigation of sets of distances associated with continuous functions.

Two metric properties of continua have been investigated in connection with some topological phenomena, and each of them can be expressed by means of a numerical quantity designed to measure the "non-arc-likeness" of a given continuum. The concept of the width was introduced by C. E. Burgess [1] in the theory of tree-like continua, and the author [7] defined the span of a metric space without any restrictions imposed upon its structure. The present paper resulted from an attempt to compare these two notions. Although, in general, the span and the width are not equal even for very primitive objects such as simple triods, some comparisons can be made and the similarities do exist (see Sections 3-5). At the end of the paper two results are obtained (see 5.3 and 5.4) concerning the set of distances between points that belong to point-inverses under continuous mappings.

1. Definitions and preliminaries. If $X$ is a non-empty metric space, we define the span $\sigma(X)$ of $X$ to be the least upper bound of the set of real numbers $\alpha$ which satisfy the following condition: there exists a connected space $C$ and continuous mappings $f_{1}, f_{2}: C \rightarrow X$ such that $f_{1}(C)=f_{2}(C)$ and $\alpha \leqslant \operatorname{dist}\left[f_{1}(c), f_{2}(c)\right]$ for $c \in C$. Equivalently (see [7], p. 209), the span $\sigma(X)$ is the least upper bound of numbers $\alpha$ for which there exist connected subsets $C_{\alpha}$ of the product $X \times X$ such that $p_{1}\left(C_{\alpha}\right)=p_{2}\left(C_{\alpha}\right)$ and $\alpha \leqslant \operatorname{dist}(x, y)$ for $(x, y) \in C_{\alpha}$, where $p_{1}$ and $p_{2}$ denote the projections of $X \times X$ onto $X$, i.e. $p_{1}(x, y)=x$ and $p_{2}(x, y)=y$ for $x, y \in X$. We note that, for compact spaces $X$, the sets $C_{\alpha}$ in the latter definition of $\sigma(X)$ can be assumed to be closed in $X \times X$.

The definition of the width is a bit more involved in that it requires rather special notation. If $A, B \subset X$ are non-empty subsets of the metric space $X$, we denote by $\delta(A)$ and $\varrho(A, B)$ the diameter of $A$ and the distance between $A$ and $B$, respectively, i.e.

$\delta(A)=\operatorname{Sup}\left\{\operatorname{dist}\left(a, a^{\prime}\right): a, a^{\prime} \in A\right\}, \quad \varrho(A, B)=\operatorname{Inf}\{\operatorname{dist}(a, b): a \in A, b \in B\}$. 1- Fundamenta Mathematicae XCVIII 
If $C$ is a collection of non-empty subsets of $X$, we denote by $|C|$ the union of all the sets which belong to $C$, and we put

$$
\operatorname{mesh}(C)=\operatorname{Sup}\{\delta(A): A \in C\} .
$$

Furthermore, we say $\boldsymbol{C}$ is a chain provided $\boldsymbol{C}$ is finite and the nerve of $\boldsymbol{C}$, if nondegenerate, is an arc (see [5], p. 318). For any collection $C$ of non-empty subsets of $X$, Chain $(C)$ will denote the family of all the chains which are contained in $\boldsymbol{C}$. For finite collections $C$ of non-empty subsets of $X$, we define real numbers $w(C)$ by the formula

$$
w(C)=\operatorname{Min}\left\{\operatorname{Max}\left\{\varrho\left(A,\left|C^{\prime}\right|\right): A \in C\right\}: C^{\prime} \in \operatorname{Chain}(C)\right\} .
$$

We also consider finite open covers of $X$ by which we mean finite collections $C$ of non-empty open subsets of $X$ such that $|C|=X$. Now, if $X$ is a non-empty compact metric space, we define the width $w(X)$ of $X$ to be the least upper bound of the set of real numbers $\alpha$ which satisfy the following condition: for each $\varepsilon>0$, there exists a finite open cover $\boldsymbol{C}$ of $X$ such that mesh $(\boldsymbol{C})<\varepsilon$ and $\alpha \leqslant w(\boldsymbol{C})$. Thus $w(X)$ is a well-defined real number and $0 \leqslant w(X) \leqslant \delta(X)$.

Let $X$ be a non-empty continuum, i.e. connected compact metric space. We say $X$ is a tree provided $X$ is homeomorphic to a connected one-dimensional polyhedron which contains no simple closed curve. A continuum $X$ is called tree-like (or arc-like) provided, for each $\varepsilon>0$, there exists a finite open cover $C$ of $X$ such that mesh $(C)<\varepsilon$ and the nerve of $C$, if non-degenerate, is a tree (or an arc, respectively). The concept of the width of a tree-like continuum was defined earlier in [1] using slightly different considerations. Before we see that, restricted to such a continuum, the two definitions coincide, we need to prove some auxiliary propositions. By a refinement of a finite open cover $C$ we mean, as usual, any finite open cover whose elements are contained in elements of $\boldsymbol{C}$.

1.1. If $\boldsymbol{C}$ is a finite open cover of a metric space and $\boldsymbol{D}$ is a refinement of $\boldsymbol{C}$ such that the nerve of $C$ is a tree and the nerve of $D$ is connected, then

$$
w(\boldsymbol{D}) \leqslant w(\boldsymbol{C})+2 \operatorname{mesh}(\boldsymbol{C}) .
$$

Proof. Let $X$ denote the space and let $C^{\prime} \in$ Chain $(\boldsymbol{C})$. The proof of 1.1 will be complete if we show the existence of a set $A \in C$ such that

$$
w(\boldsymbol{D}) \leqslant \varrho\left(A,\left|\boldsymbol{C}^{\prime}\right|\right)+2 \operatorname{mesh}(\boldsymbol{C}) .
$$

The following two cases are to be distinguished:

Case 1 . $C$ consists of at most 2 elements. Then $C$ has exactly 2 elements and let $C=\{A, B\}$. Thus $A \cap B \neq \emptyset$ and $A \cup B=|C|=X$; inequality (1) follows from

$$
w(D) \leqslant \delta(X)=\delta(A \cup B) \leqslant \delta(A)+\delta(B) \leqslant 2 \operatorname{mesh}(C) .
$$

Case 2. $C$ consists of more than 2 elements. Then the chain $C^{\prime}$ is contained in a chain $C^{\prime \prime} \subset C$ which has at least 3 elements, say $C^{\prime \prime}=\left\{C_{1}, \ldots, C_{m}\right\}$, where $m \geqslant 3$ and $C_{i} \cap C_{j} \neq \varnothing$ if and only if $|i-j| \leqslant 1$ for $i, j=1, \ldots, m$. Thus $C_{1} \cap C_{m}=\varnothing$ and the non-empty sets $C_{1}, C_{m}$ intersect some non-empty sets belonging to the refinement $\boldsymbol{D}$ of $\boldsymbol{C}$. Since the nerve of $\boldsymbol{D}$ is connected, those elements of $\boldsymbol{D}$ can be joined together by means of a chain $D^{\prime} \subset D$, so that we can write $D^{\prime}=\left\{D_{1}, \ldots, D_{n}\right\}$, where $C_{1} \cap D_{1} \neq \emptyset \neq C_{m} \cap D_{n}$ and $D_{i} \cap D_{j} \neq \varnothing$ if and only if $|i-j| \leqslant 1$ for $i, j=1, \ldots, n$. As a result, we obtain

$$
\begin{aligned}
& \varnothing \neq C_{1} \cap D_{1} \subset C_{1} \cap\left|\boldsymbol{D}^{\prime}\right|, \\
& \varnothing \neq C_{m} \cap D_{n} \subset C_{m} \cap\left|\boldsymbol{D}^{\prime}\right|,
\end{aligned}
$$

and we claim that $C_{i} \cap\left|D^{\prime}\right| \neq \varnothing$, too, for $i=2, \ldots, m-1$. Indeed, since the nerve of $C$ is a tree, all the elements of $C \backslash\left\{C_{i}\right\}(i=2, \ldots, m-1)$ can be grouped in two collections $C_{1}$ and $C_{2}$ such that $C_{1} \in C_{1}, C_{m} \in C_{2}$ and $\left|C_{1}\right| \cap\left|C_{2}\right|=\varnothing$. Supposing $C_{i} \cap\left|D^{\prime}\right|=\varnothing$, we would get

$$
\left|D^{\prime}\right| \subset\left|C \backslash\left\{C_{i}\right\}\right|=\left|C_{1}\right| \cup\left|C_{2}\right|,
$$

and since each element of $\boldsymbol{D}^{\prime}$ is contained in an element of $C$, the collection $\boldsymbol{D}^{\prime}$ would split into two collections composed of subsets of $\left|C_{1}\right|$ and $\left|C_{2}\right|$, respectively. One of these collections would contain $D_{1}$ and the other one would contain $D_{n}$. This, however, is impossible, $D^{\prime}$ being a chain; therefore $C_{i} \cap\left|\boldsymbol{D}^{\prime}\right| \neq \emptyset$ for $i=1, \ldots, m$. It follows that, for each point $y \in\left|C^{\prime \prime}\right|$, there exists an integer $i=1, \ldots, m$ and a point $y^{\prime} \in\left|D^{\prime}\right|$ such that $y, y^{\prime} \in C_{i}$, and thus

$$
\operatorname{dist}\left(y, y^{\prime}\right) \leqslant \delta\left(C_{i}\right) \leqslant \operatorname{mesh}(C) \text {. }
$$

On the other hand, $D^{\prime} \in$ Chain $(D)$ implies the existence of a set $B \in D$ satisfying the inequality

$$
w(\boldsymbol{D}) \leqslant \varrho\left(\boldsymbol{B},\left|\boldsymbol{D}^{\prime}\right|\right),
$$

and since $D$ is a refinement of $C$, there is a set $A \in C$ such that $B \subset A$. Let us select a point $x^{\prime} \in B$ and observe that $\left|\boldsymbol{C}^{\prime}\right| \subset\left|\boldsymbol{C}^{\prime \prime}\right|$. We conclude that, given arbitrary points $x \in A$ and $y \in\left|C^{\prime}\right|$, one has $\operatorname{dist}\left(x, x^{\prime}\right) \leqslant \delta(A) \leqslant \operatorname{mesh}(C)$ as well as

$$
\begin{aligned}
w(D) & \leqslant \varrho\left(B,\left|D^{\prime}\right|\right) \leqslant \operatorname{dist}\left(x^{\prime}, y^{\prime}\right) \leqslant \operatorname{dist}\left(x^{\prime}, x\right)+\operatorname{dist}(x, y)+\operatorname{dist}\left(y, y^{\prime}\right) \\
& \leqslant \operatorname{dist}(x, y)+2 \operatorname{mesh}(\boldsymbol{C}),
\end{aligned}
$$

whence (1) and the proof of 1.1 is completed.

1.2. If $X$ is a tree-like continuum and $C_{1}, C_{2}, \ldots$ is a sequence of finite open covers of $X$ such that the nerve of $C_{n}$ is a tree $(n=1,2, \ldots)$ and

$$
\operatorname{limmesh}_{n \rightarrow \infty}\left(C_{n}\right)=0 \text {, }
$$

then $\lim _{n \rightarrow \infty} w\left(C_{n}\right)=w(X)$.

Proof. Let $\gamma>0$ be a number arbitrarily chosen. If the inequality

$$
w\left(C_{n}\right)<w(X)+\gamma
$$


were violated by infinitely many positive integers $n$, the number $\alpha=w(X)+\gamma$ would have the property of possessing finite open covers $C_{n}$ of $X$ with small mesh $\left(C_{n}\right)$, by (2), and such that $\alpha \leqslant w\left(\boldsymbol{C}_{n}\right)$. According to the definition of the width $w(X)$, we would then have $\alpha \leqslant w(X)$ which contradicts the assumption that $\gamma>0$. Consequently, there exists a positive integer $n_{1}$ such that (3) holds for $n \geqslant n_{1}$.

By virtue of (2), there also exists a positive integer $n_{2}$ such that $\operatorname{mesh}\left(C_{n}\right)<\frac{1}{3} \gamma$ for $n \geqslant n_{2}$. Let $n \geqslant n_{2}$ be fixed for a while. Since $X$ is compact, there is a number $\varepsilon>0$ such that each finite open cover $C$ of $X$ with $\operatorname{mesh}(C)<\varepsilon$ is a refinement of $C_{n}$. But since $w(X)$ is the width of $X$, a cover $C$ of this type can be found satisfying the inequality

$$
w(X)-\frac{1}{3} \gamma \leqslant w(C),
$$

and the connectedness of $X$ implies the connectedness of the nerve of $C$. By 1.1, we obtain

$$
w(\boldsymbol{C}) \leqslant w\left(\boldsymbol{C}_{n}\right)+2 \operatorname{mesh}\left(\boldsymbol{C}_{n}\right)<w\left(\boldsymbol{C}_{n}\right)+\frac{2}{3} \gamma,
$$

whence $w(X)-\gamma<w\left(C_{n}\right)$. Combining the latter inequality with inequality (3), we see that

$$
\left|w\left(C_{n}\right)-w(X)\right|<\gamma
$$

provided $n \geqslant n_{1}$ and $n \geqslant n_{2}$. Thus 1.2 is proved.

Remark. It follows from 1.2 that, for tree-like continua, the width as defined in this paper is equal to that invented by Burgess [1]. On the other hand, the condition that the nerves of $C_{n}$ are trees surely implies, by (2), that the continuum $X$ from 1.2 is tree-like. It will be shown (see 3.7) that this condition cannot be omitted in 1.2.

2. The width of dendroids. Each arcwise connected tree-like continuum is said to be a dendroid. Clearly, all trees as well as all dendrites are dendroids. By a simple. triod we mean a tree which is the union of three arcs having a common end-point and being pairwise disjoint except at that point. Since chains are related to arcs in a natural way, there should also exist a relationship between the concept of the width, as defined in Section 1, and another concept that expresses a similar idea by means of arcs instead of chains. To have such a relationship, however, one should assume the existence of sufficiently many arcs in the space, and the class of dendroids seems to be an appropriate range for this purpose. Before studying them, we prove two propositions of a more general character. For any metric space $X, \operatorname{Arc}(X)$ will denote the collection of all the arcs which are contained in $X$ and of all the nonempty degenerate subsets of $X$. If $\{a\}$ is a degenerate set and $B$ is a non-empty set, we denote the distance between them by $\varrho(a, B)$ rather than by $\varrho(\{a\}, B)$.

2.1. If $X$ is a metric space, $A \in \operatorname{Arc}(X)$ and $\gamma>0$, then there exists a number $\varepsilon>0$ such that each finite open cover $\boldsymbol{C}$ of $X$ with mesh $(\boldsymbol{C})<\varepsilon$ has a chain $\boldsymbol{C}^{\prime} \in \operatorname{Chain}(\boldsymbol{C})$ satisfying the condition

$$
\operatorname{Sup}\left\{\varrho\left(a,\left|C^{\prime}\right|\right): a \in A\right\} \leqslant \gamma .
$$

Proof. If $A$ is degenerate, 2.1 tivially holds since any degenerate chain $C^{\prime}$ composed of a set $C \in C$ which contains $A$ has the distance $\varrho(A, C)=0$. Let us then assume $A$ is an arc and decompose it into $k \geqslant 3$ subarcs $A_{1}, \ldots, A_{k}$ such that $\delta\left(A_{j}\right)<\gamma$ and $A_{i} \cap A_{j} \neq \varnothing$ if and only if $|i-j| \leqslant 1$ for $i, j=1, \ldots, k$. We define

$$
\varepsilon=\frac{1}{2} \operatorname{Min}\left\{\varrho\left(A_{1} \cup \ldots \cup A_{i-1}, A_{i+1} \cup \ldots \cup A_{k}\right): i=2, \ldots, k-1\right\} .
$$

Let $C$ be a finite open cover of $X$ with mesh $(C)<\varepsilon$. The subcollection $C^{*}$ of $C$ consisting of all the elements of $C$ which meet $A$ has a connected nerve since $A$ is connected. Two elements of $C^{*}$ intersect $A_{1}$ and $A_{k}$, respectively, and thus they can be joined together by means of a chain $C^{\prime} \subset C^{*}$. Thus $A_{1} \cap\left|C^{\prime}\right| \neq \emptyset$ and $A_{k} \cap\left|C^{\prime}\right| \neq \emptyset$. We claim that $A_{i} \cap\left|C^{\prime}\right| \neq \emptyset$, too, for $i=2, \ldots, k-1$. If it were not so, the collection $C^{\prime}$ would split into two non-empty collections composed of sets which meet $A_{1} \cup \ldots \cup A_{i-1}$ and $A_{i+1} \cup \ldots \cup A_{k}$, respectively. But, $C^{\prime}$ being a chain, there would exist non-disjoint elements $C_{1}$ and $C_{2}$ belonging to these collections, respectively, and we would get

$$
\begin{aligned}
\varrho\left(A_{1} \cup \ldots \cup\right. & \left.A_{i-1}, A_{i+1} \cup \ldots \cup A_{k}\right) \\
& \leqslant \varrho\left[C_{1} \cap\left(A_{1} \cup \ldots \cup A_{i-1}\right), C_{2} \cap\left(A_{i+1} \cup \ldots \cup A_{k}\right)\right] \\
& \leqslant \delta\left(C_{1} \cup C_{2}\right) \leqslant \delta\left(C_{1}\right)+\delta\left(C_{2}\right) \leqslant 2 \operatorname{mesh}(C)<2 \varepsilon,
\end{aligned}
$$

which contradicts the definition of $\varepsilon$. Hence $\left|C^{\prime}\right|$ meets all the arcs $A_{1}, \ldots, A_{k}$. Given a point $a \in A$, there is an integer $j=1, \ldots, k$ such that $a \in A_{j}$. The condition required in 2.1 now follows from the inequalities

$$
\varrho\left(a,\left|C^{\prime}\right|\right) \leqslant \varrho\left(a, A_{j} \cap\left|C^{\prime}\right|\right) \leqslant \delta\left(A_{j}\right)<\gamma .
$$

2.2. THEOREM. If $X$ is a non-empty compact metric space, then

$$
w(X) \leqslant \operatorname{Inf}\{\operatorname{Sup}\{\varrho(x, A): x \in X\}: A \in \operatorname{Arc}(X)\} .
$$

Proof. Let $r$ stand for the real number that is the right-hand side of the latter inequality and let $\gamma>0$ be an arbitrary real number. There exists then an $A_{0} \in \operatorname{Arc}(X)$ such that

$$
\operatorname{Sup}\left\{\varrho\left(x, A_{0}\right): x \in X\right\}<r+\gamma
$$

and let $\varepsilon_{0}>0$ be the real number whose existence is guaranteed by 2.1 for $A=A_{0}$. On the other hand, it follows from the definition of the width that there is a real number $\alpha_{0}$ such that

$$
w(X)-\gamma<\alpha_{0}
$$

and, for each $\varepsilon>0$, a finite open cover $C$ of $X$ exists with mesh $(\boldsymbol{C})<\varepsilon$ and $\alpha_{0} \leqslant w(C)$, whence $w(X)<w(C)+\gamma$. In particular, for $\varepsilon=\varepsilon_{0}$, there exists such a finite open cover $\boldsymbol{C}_{0}$ and, by 2.1 , we have a chain $\boldsymbol{C}_{0}^{\prime} \in$ Chain $\left(\boldsymbol{C}_{0}\right)$ with the following property: for each point $a \in A_{0}$, there is a point $y \in\left|C_{0}^{\prime}\right|$ satisfying the condition

$$
\operatorname{dist}(a, y)<\varrho\left(a,\left|\boldsymbol{C}_{0}^{\prime}\right|\right)+\gamma \leqslant 2 \gamma \text {. }
$$


According to the definition of $w\left(C_{0}\right)$, an element $G_{0} \in C_{0}$ must exist such that

$$
w\left(\boldsymbol{C}_{0}\right) \leqslant \varrho\left(G_{0},\left|\boldsymbol{C}_{0}^{\prime}\right|\right),
$$

whence $w(X)<w\left(C_{0}\right)+\gamma \leqslant \varrho\left(G_{0},\left|C_{0}^{\prime}\right|\right)+\gamma$ and, taking a point $x_{0} \in G_{0}$, we get

$$
w(X)<\varrho\left(G_{0},\left|\boldsymbol{C}_{0}^{\prime}\right|\right)+\gamma \leqslant \varrho\left(x_{0},\left|\boldsymbol{C}_{0}^{\prime}\right|\right)+\gamma .
$$
obtain

Since to each point $a \in A_{0}$ there corresponds a point $y \in\left|C_{0}^{\prime}\right|$ fulfilling (5), we

$$
\varrho\left(x_{0},\left|C_{0}^{\prime}\right|\right) \leqslant \operatorname{dist}\left(x_{0}, y\right) \leqslant \operatorname{dist}\left(x_{0}, a\right)+\operatorname{dist}(a, y)<\operatorname{dist}\left(x_{0}, a\right)+2 \gamma,
$$

which implies that $\varrho\left(x_{0},\left|C_{0}^{\prime}\right|\right) \leqslant \varrho\left(x_{0}, A_{0}\right)+2 \gamma$, whence

$$
w(X)<\varrho\left(x_{0},\left|C_{0}^{\prime}\right|\right)+\gamma \leqslant \varrho\left(x_{0}, A_{0}\right)+3 \gamma .
$$

By (4), the width of $X$ thus satisfies the inequality $w(X)<r+4 \gamma$, which completes the proof of 2.2 .

2.3. Theorem. If $X$ is a dendroid, then

$$
w(X)=\operatorname{Inf}\{\operatorname{Sup}\{\varrho(x, A): x \in X\}: A \in \operatorname{Arc}(X)\}^{\circ} .
$$

Proof. Keeping a notation of the preceding proof, let $r$ be the number which is the right-hand side of this equality and let $\gamma>0$ be an arbitrary number. If the inequality

$$
\text { (6) } \quad w(C)<w(X)+\gamma
$$

did not hold for some finite open covers $C$ of $X$ with mesh $(C)$ as small as one wants, the number $\alpha=w(X)+\gamma$ would satisfy the condition from the definition of the width $w(X)$ and, consequently, we would have $\alpha \leqslant w(X)$ which is not the case as $\gamma>0$. Therefore there exists a number $\varepsilon_{0}>0$ such that (6) holds if $\operatorname{mesh}(C)<\varepsilon_{0}$.

The dendroid $X$ being a tree-like continuum, there exists a finite open cover $C_{0}$ of $X$ such that

$$
\operatorname{mesh}\left(C_{0}\right)<\operatorname{Min}\left\{\varepsilon_{0}, \gamma\right\}
$$

and the nerve of $C_{0}$ is either degenerate or a tree. Then, by (6), we have $w\left(\boldsymbol{C}_{0}\right)<w(X)+\gamma$. It follows from the definition of $w\left(\boldsymbol{C}_{0}\right)$ that there exists a chain $C_{0}^{\prime} \in$ Chain $\left(C_{0}\right)$ such that

$$
\operatorname{Max}\left\{\varrho\left(G,\left|C_{0}^{\prime}\right|\right): G \in C_{0}\right\}=w\left(C_{0}\right)<w(X)+\gamma,
$$

and we claim that an $A_{0} \in \operatorname{Arc}(X)$ can be picked up so that

$$
\operatorname{Sup}\left\{\varrho\left(x, A_{0}\right): x \in\left|C_{0}^{\prime}\right|\right\} \leqslant \gamma .
$$

Indeed, if $C_{0}^{\prime}$ is a degenerate chain $\{C\}$, it is enough to put $A_{0}=\left\{a_{0}\right\}$, where $a_{0} \in C$, and then $\varrho\left(x, A_{0}\right)=\operatorname{dist}\left(x, a_{0}\right) \leqslant \delta(C) \leqslant \operatorname{mesh}\left(C_{0}\right)<\gamma$ for each $x \in\left|C_{0}^{\prime}\right|=C$. If $C_{0}^{\prime}$ is non-degenerate, we have $C_{0}^{\prime}=\left\{C_{1}, \ldots, C_{k}\right\}$, where $k \geqslant 2$ and $C_{i} \cap C_{j} \neq \varnothing$ if and only if $|i-j| \leqslant 1$ for $i, j=1, \ldots, k$. Let us select two distinct points $p \in C_{1}$ and $q \in C_{k}$. Since $X$ is arcwise connected, there exists an arc $A_{0} \subset X$ joining $p$ and $q$.
Thus $C_{1} \cap A_{0} \neq \varnothing$ and $C_{k} \cap A_{0} \neq \varnothing$. We show that $C_{i} \cap A_{0} \neq \varnothing$ for $i=1, \ldots, k$. If it were not so, $k \geqslant 3$ and there would be an integer $i_{0}=2, \ldots, k-1$ such that $A_{0} \subset\left|C_{0} \backslash\left\{C_{i_{0}}\right\}\right|$. But the nerve of $C_{0}$ being a tree, all the elements of $C_{0} \backslash\left\{C_{i_{0}}\right\}$ would group in two collections $C_{1}$ and $C_{2}$ such that $C_{1} \in C_{1}, C_{k} \in C_{2}$ and $\left|C_{1}\right| \cap\left|C_{2}\right|=\varnothing$. The arc $A_{0}$ would then be contained in the union of two disjoint open sets $\left|C_{1}\right|$ and $\left|C_{2}\right|$ each of them intersecting $A_{0}$, which contradicts the connectedness of $A_{0}$. Hence $A_{0}$ meets all the sets $C_{1}, \ldots, C_{k}$. Given a point $x \in\left|C_{0}^{\prime}\right|$, there is an integer $j=1, \ldots, k$ such that $x \in C_{j}$, and (8) follows from the inequalities

$$
\varrho\left(x, A_{0}\right) \leqslant \varrho\left(x, C_{j} \cap A_{0}\right) \leqslant \delta\left(C_{j}\right) \leqslant \operatorname{mesh}\left(C_{0}\right)<\gamma .
$$

By the definition of the number $r$, there exists a point $x_{0} \in X$ such that $r-\gamma<\varrho\left(x_{0}, A_{0}\right)$. Let $G_{0} \in C_{0}$ be an element containing $x_{0}$ and let $y$ be an arbitrary point of $G_{0}$. Clearly, to each point $x \in\left|C_{0}^{\prime}\right|$, there corresponds a point $a \in A_{0}$ with $\operatorname{dist}(x, a)<\varrho\left(x, A_{0}\right)+\gamma$, whence $\operatorname{dist}(x, a)<2 \gamma$, according to (8). As a result we obtain

$$
\begin{aligned}
r & <\varrho\left(x_{0}, A_{0}\right)+\gamma \leqslant \operatorname{dist}\left(x_{0}, a\right)+\gamma \\
& \leqslant \operatorname{dist}\left(x_{0}, y\right)+\operatorname{dist}(y, x)+\operatorname{dist}(x, a)+\gamma \\
& <\delta\left(G_{0}\right)+\operatorname{dist}(y, x)+3 \gamma \\
& \leqslant \operatorname{mesh}\left(C_{0}\right)+\operatorname{dist}(y, x)+3 \gamma<\operatorname{dist}(y, x)+4 \gamma,
\end{aligned}
$$

which implies that $r-4 \gamma \leqslant \varrho\left(G_{0},\left|C_{0}^{\prime}\right|\right)<w(X)+\gamma$, by (7). Thus $r-5 \gamma<w(X)$ and, $\gamma$ being an arbitrarily taken positive number, we have $r \leqslant w(X)$, whence $w(X)=r$, by 2.2. The proof of 2.3 is complete.

2.4. If $X=A_{0} \cup A_{1} \cup A_{2}$ is a simple triod, where $A_{i}$ are arcs having a common end-point $v$ and $A_{i} \cap A_{i+1}=\{v\}$ for $i=0,1,2$ and the subscripts of $A_{j}$ taken mod3, then

$$
w(X)=\operatorname{Min}\left\{\operatorname{Max}\left\{\varrho\left(x, A_{i+1} \cup A_{i+2}\right): x \in A_{i}\right\}: i=0,1,2\right\} .
$$

Proof. Let $m$ stand for this Min Max. It follows from 2.2 that $w(X) \leqslant m$. If $A \in \operatorname{Arc}(X)$, there is a subscript $i=0,1,2$ such that $A \subset A_{i+1} \cup A_{i+2}$, whence

$$
\varrho\left(x, A_{i+1} \cup A_{i+2}\right) \leqslant \varrho(x, A)
$$

for each point $x \in X$. Consequently, we get the inequalities

$$
m \leqslant \operatorname{Max}\left\{\varrho\left(x, A_{i+1} \cup A_{i+2}\right): x \in A_{i}\right\} \leqslant \operatorname{Sup}\{\varrho(x, A): x \in X\},
$$

and 2.3 implies $m \leqslant w(X)$. Thus $w(X)=m$.

3. Some continuity properties. The span and the width, when treated as realvalued functions defined on collections of tree-like continua, seem to behave alike as far as their continuity is concerned. If $X$ is a non-empty subset of a metric space $Z$ and $\varepsilon>0$, we call the set $\{z \in Z: \varrho(z, X)<\varepsilon\}$ the $\varepsilon$-neighbourhood of $X$ in $Z$.

3.1. Let $X$ be a non-empty compact set contained in a metric space $Z$. If $\beta$ is a real number and, for $n=1,2, \ldots$, there exists a subset $Z_{n}$ of the $(1 / n)$-neighbourhood of $X$ in $Z$ with $\beta \leqslant \sigma\left(Z_{n}\right)$, then $\beta \leqslant \sigma(X)$ (compare [7], p. 211). 
Proof. Denote by $p_{1}, p_{2}: Z \times Z \rightarrow Z$ the standard projections of the product $Z \times Z$ onto $Z$. Let $\gamma>0$ be an arbitrary real number. Since $\beta-\gamma<\sigma\left(Z_{n}\right)$, it follows from the definition of the span that there exists a non-empty connected set $C_{n} \subset Z_{n} \times Z_{n}$ such that $p_{1}\left(C_{n}\right)=p_{2}\left(C_{n}\right)$ and $\beta-\gamma \leqslant \operatorname{dist}(x, y)$ for $(x, y) \in C_{n}(n=1,2, \ldots)$. Let us select points $c_{n} \in C_{n}$ and observe that the set $Z_{n} \times Z_{n}$, hence also the set $C_{n}$, is contained in the $(\sqrt{2} / n)$-neighbourhood of $X \times X$ in $Z \times Z$. Consequently, there exist points $z_{n} \in X \times X$ such that dist $\left(c_{n}, z_{n}\right)<\sqrt{2} / n$ for $n=1,2, \ldots$ By the compactness of $X \times X$, a subsequence $z_{n_{1}}, z_{n_{2}}, \ldots$ (where $n_{1}<n_{2}<\ldots$ ) converges to a point $z \in X \times X$, whence also

(9)

$$
\lim _{i \rightarrow \infty} c_{n_{t}}=z .
$$

We define $D=\mathrm{Ls}_{i \rightarrow \infty} C_{n_{i}}$ (see [5], p. 337). Thus each point of $D$ is the limit of a sequence of points of $C_{n_{1}} \cup C_{n_{2}} \cup \ldots$ It follows that $\beta-\gamma \leqslant \operatorname{dist}(x, y)$ for $(x, y) \in D$. Moreover, given a point $d \in D$, there exist points $u_{j} \in C_{n_{i_{j}}}$ (where $i_{1}<i_{2}<\ldots$ ) such that the sequence $u_{1}, u_{2}, \ldots$ converges to $d$. The point $u_{j}$ belongs to the $\left(\sqrt{2} / n_{i_{j}}\right)$-neighbourhood of $X \times X$, whence $d \in X \times X$, so that $D \subset X \times X$. On the other hand, since $p_{1}\left(C_{n_{i j}}\right)=p_{2}\left(C_{n_{i_{j}}}\right)$, there are points $c_{j}^{\prime} \in C_{n_{i_{j}}}$ such that $p_{1}\left(u_{j}\right)=p_{2}\left(c_{j}^{\prime}\right)$. We conclude, as we did before for the points $c_{n}$, that a subsequence $c_{j_{1}}^{\prime}, c_{j_{2}}^{\prime}, \ldots$ (where $j_{1}<j_{2}<\ldots$ ) converges to a point $z^{\prime} \in X \times X$. By the definition of $D$, we have $z^{\prime} \in D$, and

$$
p_{1}(d)=\lim _{k \rightarrow \infty} p_{1}\left(u_{j_{k}}\right)=\lim _{k \rightarrow \infty} p_{2}\left(c_{j_{k}}^{\prime}\right)=p_{2}\left(z^{\prime}\right),
$$

which implies that $p_{1}(D) \subset p_{2}(D)$. A symmetric argument shows that $p_{2}(D) \subset p_{1}(D)$, whence $p_{1}(D)=p_{2}(D)$.

Now, we claim that the set $D$ is connected. Suppose it is not, and notice that $z \in D$, by (9). Then there exist two disjoint open subsets $U$ and $V$ of $Z \times Z$ such that $D \subset U \cup V, z \in U$ and $D \cap V \neq \varnothing$. Let $d^{\prime} \in D \cap V$ be a point. As we have seen, there are points $v_{m} \in C_{n_{l_{m}}}$ (where $l_{1}<l_{2}<\ldots$ ) which converge to $d^{\prime}$, so that $v_{m} \in V$ for $m \geqslant m_{1}$, where $m_{1}$ is a positive integer. By (9), $c_{n_{l_{m}}} \in U$ for $m \geqslant m_{2}$. The connected set $C_{n_{I_{m}}}$ intersects both $U$ and $V$ provided $m \geqslant m_{1}$ and $m \geqslant m_{2}$. Therefore, almost all of the sets $C_{n_{l}}$ contain points $c_{m}^{\prime \prime} \notin U \cup V$ which, again, must have a subsequence converging to a point $z^{\prime \prime} \in D$. This implies that $z^{\prime \prime} \notin U \cup V$, contrary to the inclusion $D \subset U \cup V$. Hence $D$ is a connected set.

Setting $\alpha=\beta-\gamma$ and $C_{\alpha}=D$ in the definition of $\sigma(X)$, we obtain $\beta-\gamma \leqslant \sigma(X)$. Since $\gamma$ was an arbitrarily chosen positive number, the inequality $\beta \leqslant \sigma(X)$ follows and the proof of 3.1 is completed.

If $X$ is a non-empty subset of a metric space $Z$ and $\varepsilon>0$, we say that a continuous mapping $f: X \rightarrow Z$ is an $\varepsilon$-translation provided dist $[x, f(x)]<\varepsilon$ for $x \in X$. Thus if $f: X \rightarrow Z$ is an $\varepsilon$-translation, then the set $f(X)$ is contained in the $\varepsilon$-neighbourhood of $X$ in $Z$. It follows directly from the triangle inequality for distances that if $f: X \rightarrow Z$ is an $\varepsilon$-translation, then

$$
|\operatorname{dist}(x, y)-\operatorname{dist}[f(x), f(y)]|<2 \varepsilon \quad(x, y \in X) .
$$

3.2. Let $X$ be a non-empty set contained in a metric space $Z$. If $\beta$ is a real number and, for $n=1,2, \ldots$, there exists $a(1 / n)$-translation $f_{n}: X \rightarrow Z$ such that $\sigma\left[f_{n}(X)\right] \leqslant \beta$, then $\sigma\left(X^{\prime}\right) \leqslant \beta$.

Proof. Assume $\alpha$ is a real number and $C \subset X \times X$ is a connected set such that $p_{1}(C)=p_{2}(C)$ and $\alpha \leqslant \operatorname{dist}(x, y)$ for $(x, y) \in C$, where $p_{1}$ and $p_{2}$ are the standard projections of $Z \times Z$ onto $Z$. The set

$$
C_{n}=\left\{\left(f_{n}(x), f_{n}(y)\right):(x, y) \in C\right\}
$$

is connected and $C_{n} \subset f_{n}(X) \times f_{n}(X)$. Moreover,

$$
p_{1}\left(C_{n}\right)=f_{n}\left[p_{1}(C)\right]=f_{n}\left[p_{2}(C)\right]=p_{2}\left(C_{n}\right)
$$

and it follows from (10) that, for any point $\left(f_{n}(x), f_{n}(y)\right)$ of $C_{n}$, we have

$$
\alpha-2 / n \leqslant \operatorname{dist}(x, y)-2 / n<\operatorname{dist}\left[f_{n}(x), f_{n}(y)\right],
$$

whence $\alpha-2 / n \leqslant \sigma\left[f_{n}(X)\right]$. Consequently, the inequality $\alpha-2 / n \leqslant \beta$ holds for $n=1,2, \ldots$ This means that $\alpha \leqslant \beta$ and, as a result, the least upper bound $\sigma(X)$ of such numbers $\alpha$ also satisfies the inequality $\sigma(X) \leqslant \beta$.

3.3. LeMMa. Let $X$ be a non-empty subset of a bounded metric space $Z$ and let $f: X \rightarrow Z$ be an $\varepsilon$-translation. If $C$ is a finite open cover of $f(X)$ and $C^{\prime}=\left\{f^{-1}(G): G \in C\right\}$, then

$$
\left|\operatorname{mesh}(\boldsymbol{C})-\operatorname{mesh}\left(\boldsymbol{C}^{\prime}\right)\right| \leqslant 2 \varepsilon, \quad\left|w(\boldsymbol{C})-w\left(\boldsymbol{C}^{\prime}\right)\right| \leqslant 2 \varepsilon .
$$

Proof. By (10), for any pair of non-empty sets $A, B \subset f(X)$, we get the inequalities

$$
\left|\delta(A)-\delta\left[f^{-1}(A)\right]\right| \leqslant 2 \varepsilon, \quad\left|\varrho(A, B)-\varrho\left[f^{-1}(A), f^{-1}(B)\right]\right| \leqslant 2 \varepsilon,
$$

which imply the two inequalities required in 3.3 , respectively. It suffices to notice that some sets $G_{1}, \ldots, G_{n}$ of $C$ form a chain if and only if the sets $f^{-1}\left(G_{1}\right), \ldots, f^{-1}\left(G_{n}\right)$ form a chain (in $C^{\prime}$ ), and

$$
f^{-1}\left(G_{1} \cup \ldots \cup G_{n}\right)=f^{-1}\left(G_{1}\right) \cup \ldots \cup f^{-1}\left(G_{n}\right) .
$$

3.4. Let $X$ be a non-empty compact set contained in a metric space $Z$. If $\beta$ is a real number and, for $n=1,2, \ldots$, there exists a $(1 / n)$-translation $f_{n}: X \rightarrow Z$ such that $\beta \leqslant w\left[f_{n}(X)\right]$, then $\beta \leqslant w(X)$.

Proof. Since $\beta-1 / n<w\left[f_{n}(X)\right](n=1,2, \ldots)$, it follows from the definition of the width that there exists a finite open cover $C_{n}$ of $f_{n}(X)$ such that

$$
\operatorname{mesh}\left(\boldsymbol{C}_{n}\right)<1 / n, \quad \beta-1 / n \leqslant w\left(\boldsymbol{C}_{n}\right) .
$$

We take the finite open cover $C_{n}^{\prime}=\left\{f_{n}^{-1}(G): G \in C_{n}\right\}$ of $X(n=1,2, \ldots)$ which, according to 3.3 , fulfills the conditions

$$
\operatorname{mesh}\left(C_{n}^{\prime}\right) \leqslant \operatorname{mesh}\left(C_{n}\right)+2 / n<3 / n
$$


and $\beta-3 / n \leqslant w\left(C_{n}\right)-2 / n \leqslant w\left(C_{n}^{\prime}\right)$. Setting $\alpha=\beta-3 / n$ and $\boldsymbol{C}=\boldsymbol{C}_{m}^{\prime}(m \geqslant n)$ in the definition of $w(X)$, we obtain $\beta-3 / n \leqslant w(X)$, by (11). The inequality $\beta \leqslant w(X)$ now follows.

3.5. Let $X$ be a tree-like continuum contained in a metric space $Z$. If, $\beta$ is a real number and, for $n=1,2, \ldots$, there exists a $(1 / n)$-translation $f_{n}: X \rightarrow Z$ such that $Z_{n}=f_{n}(X)$ is a tree-like continuum with $w\left(Z_{n}\right) \leqslant \beta$, then $w(X) \leqslant \beta$.

Proof. We can assume $X$ is non-degenerate; otherwise $w(X)=0$. Thus all but a finite number of the continua $Z_{n}$ also are non-degenerate and, without loss of generality, we can as well assume that each $Z_{n}$ is a non-degenerate tree-like continuum $(n=1,2, \ldots)$. According to 1.2 , there exists a finite open cover $C_{n}$ of $Z_{n}$ $(n=1,2, \ldots)$ such that the nerve of $C_{n}$ is a tree and

$$
\operatorname{mesh}\left(C_{n}\right)<1 / n, \quad\left|w\left(C_{n}\right)-w\left(Z_{n}\right)\right|<1 / n .
$$

The finite open cover $C_{n}^{\prime}$ of $X$ from the proof of 3.4 fulfills condition (11), by 3.3. Furthermore, for $n=1,2, \ldots$, we have

$$
w\left(C_{n}^{\prime}\right) \leqslant w\left(C_{n}\right)+2 / n<w\left(Z_{n}\right)+3 / n \leqslant \beta+3 / n,
$$

and the nerve of $C_{n}^{\prime}$ is obviously the same as the nerve of $C_{n}$; thus it is a tree. Since (11) implies (2) with $\boldsymbol{C}_{n}$ replaced by $\boldsymbol{C}_{n}^{\prime}$, we can apply 1.2 again to conclude from (12) that

$$
w(X)=\lim _{n \rightarrow \infty} w\left(C_{n}^{\prime}\right) \leqslant \lim _{n \rightarrow \infty}(\beta+3 / n)=\beta .
$$

Remark. Obserye that the tree-likeness of $Z_{n}$ in 3.5 implies, by 3.3, the treelikeness of the continuum $X$ itself. However, even if the continuum $X$ is assumed to be tree-like, the conclusion of 3.5 is no longer true when the tree-likeness of the continua $Z_{n}$ is dropped. We provide an example to explain this possibility (see 3.7).

3.6. Corollary. If $X$ is a tree-like continuum contained in a metric space and, for $n=1,2, \ldots$, a tree-like continum $X_{n}$ is the image of $X$ under an $\varepsilon_{n}$-translation such that

$$
\lim _{n \rightarrow \infty} \varepsilon_{n}=0
$$

then $\lim \sigma\left(X_{n}\right)=\sigma(X)$ and $\lim _{n \rightarrow \infty} w\left(X_{n}\right)=w(X)$.

$\lim _{n \rightarrow \infty} \sigma\left(X_{n}\right)=\sigma(X)$ and $\lim _{n \rightarrow \infty}$

3.7. EXAMPLE. There exists a simple triod $T$ on the plane $R^{2},(1 / n)$-translations $f_{n}: T \rightarrow R^{2}$ and finite open covers $C_{n}$ of $T(n=1,2, \ldots)$ such that
(i) $w(T)=1$,
(ii) $w\left[f_{n}(T)\right]=0$ for $n=1,2, \ldots$, and
(iii) $\lim _{n \rightarrow \infty} \operatorname{mesh}\left(C_{n}\right)=\lim _{n \rightarrow \infty} w\left(C_{n}\right)=0$.

$\underset{n \rightarrow \infty}{n \rightarrow \infty}, \quad S_{n \rightarrow \infty}$

Proof. Let $S_{0}, S_{1}$ and $S_{2}$ be the straight-line segments joining the origin with the points $(0,1),(1,0)$ and $(0,-1)$, respectively. Then $T=S_{0} \cup S_{1} \cup S_{2}$ is a simple triod and (i) follows from 2.4. Let $f_{n}: T \rightarrow R^{2}$ be a $(1 / n)$-translation such that $f_{n}(T)$ is topologically a disk $(n=1,2, \ldots)$. We get (ii), by 2.2 , which means that if $D$ is a finite open cover of the $\operatorname{disk} f_{n}(T)$ with $\operatorname{mesh}(D)$ sufficiently small (depending on $n$ ), then $w(\boldsymbol{D})$ is small, too. Thus there exists, for $n=1,2, \ldots$, a finite open cover $\boldsymbol{D}_{\boldsymbol{n}}$ of $f_{n}(T)$ such that

$$
\operatorname{mesh}\left(D_{n}\right)<1 / n, \quad w\left(D_{n}\right)<1 / n .
$$

We define $C_{n}=\left\{f_{n}^{-1}(G): G \in D_{n}\right\}(n=1,2, \ldots)$. It follows from 3.3 that $\boldsymbol{C s}$ satisfy condition (iii).

4. Simple triods and trees. The results of this section indicate that the problem of finding a relationship between the span and the width of tree-like continua reduces partially to another problem, rather combinatorial in its nature, namely that of connecting the width of a tree with the widths of simple triods contained in it.

4.1. If $T$ is a simple triod, then $w(T) \leqslant \sigma(T)$.

Proof. By 2.4, this is a consequence of a lemma proved in [8].

Remark. The inequality in 4.1 cannot be replaced by the equality (see 4.5 ). Also, the assumption that $T$ is a simple triod is essential in 4.1. To support the latter statement, we study, in 4.2 below, some properties of an example of a tree which has been constructed in [9].

4.2. EXAMPLE. There exists a tree $T$ in the 3-space $R^{3}$ such that

(i) $w(T)=1$,

(ii) $\sigma(T)=\frac{1}{2}$, and

(iii) $T=A_{0} \cup A_{1} \cup A_{2} \cup A_{3}$, where $A_{i}$ are arcs having a common end-point $v$ and $A_{i} \cap A_{j}=\{v\}$ for $i \neq j(i, j=0,1,2,3)$; thus $T$ is a simple " 4 -od".

Proof. The space $R^{3}$ with the ordinary Pythagorean distance will be used. Given two points $p, q \in R^{3}$, we denote by $\overline{p q}$ the straight-line segment having $p$ and $q$ as the end-points. For $i=1,2,3$, we take the points

$$
p_{i}=\left(\frac{1}{2} \cos \frac{2}{3} \pi i, \frac{1}{2} \sin \frac{2}{3} \pi i, 0\right), \quad q_{i}=\left(\cos \frac{2}{3} \pi(i+1), \sin \frac{2}{3} \pi(i+1), 0\right),
$$

and let $q_{0}=(0,0,1)$ and $v=(0,0,0)$. We define $A_{0}=\overline{q_{0} v}$ and $A_{i}=\overline{p_{i} q_{i}} \cup \overline{p_{i} v}$ $(i=1,2,3)$. Then the union $T$ of these arcs satisfies condition (iii). We also denote

$$
B=A_{1} \cup A_{2} \cup A_{3},
$$

so that $B$ is a simple triod and $T=A_{0} \cup B$. Clearly, $\operatorname{dist}(x, v) \leqslant 1$ for $x \in B$. Since $v \in A_{0}$, we get

$$
\operatorname{Sup}\left\{\varrho\left(x, A_{0}\right): x \in T\right\} \leqslant 1,
$$

which implies that $w(T) \leqslant 1$, by 2.2. Now, let $A \in \operatorname{Arc}(T)$. If $A \subset B$, we have

$$
1=\operatorname{dist}\left(q_{0}, v\right)=\varrho\left(q_{0}, B\right) \leqslant \varrho\left(q_{0}, A\right),
$$

whence

$$
1 \leqslant \operatorname{Sup}\{\varrho(x, A): x \in T\}
$$


If $A \not \subset B$, there is a subscript $j=1,2,3$ such that $A \subset A_{n} \cup A_{j}$. The set $A_{0} \cup A_{j}$ is contained in the cylindrical section

$$
S=\left\{(r \cos \theta, r \sin \theta, t): 0 \leqslant r \leqslant 1, \frac{2}{3} \pi j \leqslant \theta \leqslant \frac{2}{3} \pi(j+1), 0 \leqslant t \leqslant 1\right\}
$$

and one of the points $q_{i}$, namely the point

$$
q=\left(\cos \frac{2}{3} \pi(j+2), \sin \frac{2}{3} \pi(j+2), 0\right)
$$

has the distance $\varrho(q, S)=1$. Since $A \subset A_{0} \cup A_{j} \subset S$, we obtain $\varrho(q, S) \leqslant \varrho(q, A)$, whence (13) holds again. By 2.3 , this implies the inequality $1 \leqslant w(T)$, and so (i) is proved.

The set $T^{\prime}=\overline{p_{1} v} \cup \overline{p_{2} v} \cup \overline{p_{3} v}$ is a simple triod contained in $T$, and $w\left(T^{\prime}\right)=\frac{1}{2}$, by 2.4. It follows from 4.1 that $\frac{1}{2} \leqslant \sigma\left(T^{\prime}\right) \leqslant \sigma(T)$. Thus, to complete the proof of (ii), we have to show that $\sigma(T) \leqslant \frac{1}{2}$. Suppose, on the contrary, that $\sigma(T)$ exceeds $\frac{1}{2}$. Then there exists a number $\alpha_{0}>\frac{1}{2}$, a continuum $C$ and two continuous mappings $f_{1}, f_{2}: C \rightarrow T$ such that $f_{1}(C)=f_{2}(C)$ and $\alpha_{0} \leqslant \operatorname{dist}\left[f_{1}(c), f_{2}(c)\right]$ for $c \in C$. The vertex $v$ is a cut-point of $T$. If $v \notin f_{1}(C)$, the continuum $f_{1}(C)$ would be contained in one of the sets $A_{i} \backslash\{v\}(i=0,1,2,3)$, which is impossible since the span of any arc is zero. Thus $v \in f_{1}(C)$. We denote

$$
V_{i}=f_{i}^{-1}(v), \quad X_{i}=f_{i}^{-1}\left(A_{0}\right), \quad Y_{i}=f_{i}^{-1}(B) \quad(i=1,2) .
$$

Let $\leqslant \leqslant_{0}$ be the ordering of the arc $A_{0}$ from $v$ to $q_{0}$, that is $(0,0, t) \leqslant \leqslant_{0}\left(0,0, t^{\prime}\right)$ if and only if $t \leqslant t^{\prime}$. The sets

$$
P_{1}=\left\{c \in X_{1} \cap X_{2}: f_{1}(c) \leqslant_{0} f_{2}(c)\right\}, \quad P_{2}=\left\{c \in X_{1} \cap X_{2}: f_{2}(c) \leqslant_{0} f_{1}(c)\right\}
$$

are compact subsets of $C$ whose union is $X_{1} \cap X_{2}$. They are also disjoint since $f_{1}(c) \neq f_{2}(c)$ for $c \in C$. Hence

(15) $X_{1} \cap X_{2}=P_{1} \cup P_{2}, \quad P_{1} \cap P_{2}=\varnothing, \quad V_{i} \cap X_{1} \cap X_{2} \subset P_{i} \quad(i=1,2)$.

We claim that a decomposition similar to (15) also exists for the set $Y_{1} \cap Y_{2}$. More precisely, we are going to prove that there exist compact sets $Q_{1}$ and $Q_{2}$ satisfying the conditions

(16) $Y_{1} \cap Y_{2}=Q_{1} \cup Q_{2}, \quad Q_{1} \cap Q_{2}=\varnothing, \quad V_{i} \cap Y_{1} \cap Y_{2} \subset Q_{i} \quad(i=1,2)$, or, in other words, that the set $Y_{1} \cap Y_{2}$ is not connected between $V_{1} \cap Y_{1} \cap Y_{2}$ and $V_{2} \cap Y_{1} \cap Y_{2}$. This will be achieved when we show that the set $Y_{1} \cap Y_{2}$ is not connected between any two points belonging to these sets (see [6], p. 168). Let $d_{i} \in V_{i} \cap Y_{1} \cap Y_{2}(i=1,2)$ be points arbitrarily selected. By $(14)$, we have $f_{1}\left(d_{1}\right)=v=f_{2}\left(d_{2}\right)$, whence $f_{2}\left(d_{1}\right) \neq v \neq f_{1}\left(d_{2}\right)$. Also by (14), the points $f_{1}\left(d_{2}\right)$ and $f_{2}\left(d_{1}\right)$ are in $B$, so that each of them is in one of the sets $A_{i} \backslash\{v\}(i=1,2,3)$. Thus there exists a subscript $k=1,2,3$ such that the arc $A_{k}$ contains neither $f_{1}\left(d_{2}\right)$ nor $f_{2}\left(d_{1}\right)$. Let $l$ and $m$ be the two remaining subscripts from $\{1,2,3\}$ arranged so that the arcs $A_{l}$ and $A_{m}$ are obtained from $A_{k}$ by the counter-clockwise rotation through the angles $\frac{2}{3} \pi$ and $\frac{4}{3} \pi$, respectively. In other words, $(k, l, m)$ is either $(1,2,3)$ or $(2,3,1)$ or $(3,1,2)$. We consider a retraction $g: B \rightarrow A_{k} \cup A_{m}$ which is defined in the following way. The mapping $g$ maps $A_{l}$ into $A_{m}, g\left(p_{l}\right)=v, g\left(q_{l}\right)=p_{m}$, and $g$ is linear on both segments $\overline{p_{l} q_{l}}$ and $\overline{p_{l} v}$ whose union is $A_{l}$. Consequently, we have

$$
g^{-1}(x)=\left\{\begin{array}{lll}
\{x\} & \text { for } & x \in\left(A_{k} \cup A_{m}\right) \backslash \overline{p_{m} v}, \\
p_{l} v & \text { for } & x=v, \\
\{x, y(x)\} & \text { for } & x \in \overline{p_{m} v} \backslash\{v\},
\end{array}\right.
$$

where $y(x)$ is the point of the segment $\overline{p_{l} q_{l}}$ such that

$$
\frac{\operatorname{dist}\left[p_{l}, y(x)\right]}{\operatorname{dist}\left(p_{l}, q_{l}\right)}=\frac{\operatorname{dist}(v, x)}{\operatorname{dist}\left(v, p_{m}\right)} \text {. }
$$

Next, we need to show that

$$
\delta\left[g^{-1}(x)\right] \leqslant \frac{1}{2} \quad\left(x \in A_{k} \cup A_{m}\right) .
$$

Since $\delta\left(\overline{p_{l} v}\right)=\operatorname{dist}\left(p_{l}, v\right)=\frac{1}{2}$, inequality (19) holds for $x \in\left(A_{k} \cup A_{m}\right) \backslash \overline{p_{m} v}$ or $x=v$, by (17). Assume then that $x$ is a point of $\overline{p_{m} v}$ and $x \neq v$. It has to be shown that dist $[x, y(x)] \leqslant \frac{1}{2}$ for such $x \in \overline{p_{m} v} \backslash\{v\}$. To this end, let us denote $\lambda=\operatorname{dist}(v, x)$, and observe that $\operatorname{dist}\left(p_{l}, q_{l}\right)=\frac{1}{2} \sqrt{7}$ and $\operatorname{dist}\left(v, p_{m}\right)=\frac{1}{2}$. Hence $0 \leqslant \lambda \leqslant \frac{1}{2}$ and

$$
\operatorname{dist}\left[p_{l}, y(x)\right]=\lambda \sqrt{7},
$$

by (18). Also, it follows from the definition of $l$ and $m$ that $p_{m}$ is the mid-point of the segment $\overline{q_{l} v}$. Thus the point $x$ belongs to $\overline{q_{l} v}$ and

$$
\operatorname{dist}\left(q_{l}, x\right)=\operatorname{dist}\left(q_{l}, v\right)-\operatorname{dist}(v, x)=1-\lambda .
$$

On the other hand, we get

$$
\operatorname{dist}\left[q_{l}, y(x)\right]=\operatorname{dist}\left(q_{l}, p_{l}\right)-\operatorname{dist}\left[p_{l}, y(x)\right]=\sqrt{7}\left(\frac{1}{2}-\lambda\right),
$$

and $\cos \theta=\frac{5}{14} \sqrt{7}$, where $\theta$ is the angle between the segments $\overline{q_{l} v}$ and $\overline{q_{l} p_{l}}$. As a result, we obtain

$$
\begin{aligned}
(\operatorname{dist}[x, y(x)])^{2} & =(1-\lambda)^{2}+7\left(\frac{1}{2}-\lambda\right)^{2}-2 \sqrt{7}(1-\lambda)\left(\frac{1}{2}-\lambda\right) \cos \theta \\
& =(1-\lambda)^{2}+7\left(\frac{1}{2}-\lambda\right)^{2}-5(1-\lambda)\left(\frac{1}{2}-\lambda\right) \\
& =\frac{1}{4}+3 \lambda\left(\lambda-\frac{1}{2}\right) \leqslant \frac{1}{4},
\end{aligned}
$$

whence dist $[x, y(x)] \leqslant \frac{1}{2}$ and, by (17), the proof of (19) is complete.

Let $\leqslant_{k}$ be the ordering of the arc $A_{k} \cup A_{m}$ from $q_{k}$ to $q_{m}$, the end-points of this arc. The sets

$$
\begin{aligned}
& Q_{1}^{\prime}=\left\{c \in Y_{1} \cap Y_{2}: g f_{1}(c) \leqslant_{k} g f_{2}(c)\right\}, \\
& Q_{2}^{\prime}=\left\{c \in Y_{1} \cap Y_{2}: g f_{2}(c) \leqslant_{k} g f_{1}(c)\right\}
\end{aligned}
$$

are compact subsets of $C$ whose union is $Y_{1} \cap Y_{2}$, by (14). Since $\frac{1}{2}<\alpha_{0} \leqslant \operatorname{dist}\left[f_{1}(c), f_{2}(c)\right]$ for $c \in C$, it follows from (19) that $g f_{1}(c) \neq g f_{2}(c)$ for 
$c \in Y_{1} \cap Y_{2}$. Therefore the sets $Q_{1}^{\prime}$ and $Q_{2}^{\prime}$ are disjoint. Moreover, the points $f_{1}\left(d_{2}\right)$ and $f_{2}\left(d_{1}\right)$ both belong to $A_{l} \cup A_{m}$, whence

$$
g f_{1}\left(d_{2}\right), g f_{2}\left(d_{1}\right) \in g\left(A_{l} \cup A_{m}\right)=A_{m},
$$

and because $g f_{i}\left(d_{i}\right)=g(v)=v(i=1,2)$, we conclude that

$$
g f_{1}\left(d_{1}\right)=v \leqslant_{k} g f_{2}\left(d_{1}\right), \quad g f_{2}\left(d_{2}\right)=v \leqslant_{k} g f_{1}\left(d_{2}\right),
$$

which means that $d_{i} \in Q_{i}^{\prime}(i=1,2)$. The decomposition of $Y_{1} \cap Y_{2}$ into $Q_{1}^{\prime}$ and $Q_{2}^{\prime}$ then establishes the non-connectedness of $Y_{1} \cap Y_{2}$ between $d_{1}$ and $d_{2}$. Consequently, we have also proved the existence of compact sets $Q_{1}$ and $Q_{2}$ which satisfy (16).

We now distinguish two cases to prove that

$$
X_{1} \cap Y_{2} \neq \varnothing \neq X_{2} \cap Y_{1} \text {. }
$$

Let $u=\left(0,0, \frac{1}{2}\right)$.

Case 1. $u \in f_{1}(C)$. Since $f_{1}(C)=f_{2}(C)$, there exist points $c_{i} \in C$ such that $f_{i}\left(c_{i}\right)=u(i=1,2)$. But $u \in A_{0}$, so that $c_{i} \in X_{i}(i=1,2)$, by (14). Each point of $T$ whose distance from $u$ exceeds $\frac{1}{2}$ belongs to $B$. It follows from the inequalities

$$
\frac{1}{2}<\alpha_{0} \leqslant \operatorname{dist}\left[f_{1}\left(c_{i}\right), f_{2}\left(c_{i}\right)\right] \quad(i=1,2)
$$

that $f_{2}\left(c_{1}\right), f_{1}\left(c_{2}\right) \in B$, whence $c_{1} \in Y_{2}$ and $c_{2} \in Y_{1}$, by (14). We get $c_{1} \in X_{1} \cap Y_{2}$ and $c_{2} \in X_{2} \cap Y_{1}$.

Case 2. $u \notin f_{1}(C)$. We know that $v \in f_{1}(C)$. Let $w$ be the last point of the segment $A_{0}$ which belongs to $f_{1}(C)$, in the ordering $\leqslant_{0}$. The set $A_{0} \cap f_{1}(C)$ is connected, $f_{1}(C)$ being a continuum. Thus, in this case, we have $w \in \overline{u v}$. Consequently, by the definition of $w$, each point of $f_{1}(C)$ whose distance from $w$ exceeds $\frac{1}{2}$ must belong to $B$. Since $f_{1}(C)=f_{2}(C)$, there exist points $c_{i}^{\prime} \in C$ such that $f_{i}\left(c_{i}^{\prime}\right)=w$ $(i=1,2)$. As in Case 1 , we get $c_{1}^{\prime} \in X_{1} \cap Y_{2}$ and $c_{2}^{\prime} \in X_{2} \cap Y_{1}$, which completes the proof of (20).

The sets

$$
M=P_{1} \cup Q_{2} \cup\left(X_{2} \cap Y_{1}\right), \quad N=P_{2} \cup Q_{1} \cup\left(X_{1} \cap Y_{2}\right)
$$

are compact and non-empty, by (20). Also, we have

$$
\begin{aligned}
C & =C \cap C=f_{1}^{-1}(T) \cap f_{2}^{-1}(T)=f_{1}^{-1}\left(A_{0} \cup B\right) \cap f_{2}^{-1}\left(A_{0} \cup B\right) \\
& =\left(X_{1} \cup Y_{1}\right) \cap\left(X_{2} \cup Y_{2}\right)=\left(X_{1} \cap X_{2}\right) \cup\left(X_{1} \cap Y_{2}\right) \cup\left(X_{2} \cap Y_{1}\right) \cup\left(Y_{1} \cap Y_{2}\right) \\
& =P_{1} \cup P_{2} \cup\left(X_{1} \cap Y_{2}\right) \cup\left(X_{2} \cap Y_{1}\right) \cup Q_{1} \cup Q_{2}=M \cup N,
\end{aligned}
$$

by (14), (15) and (16). Since $A_{0} \cap B=\{v\}$, it follows from (14) that $X_{i} \cap Y_{i}=V_{i}$ $(i=1,2)$. Hence

$$
\begin{aligned}
M \cap N= & \left(P_{1} \cap Q_{1}\right) \cup\left(P_{1} \cap X_{1} \cap Y_{2}\right) \cup\left(Q_{2} \cap P_{2}\right) \cup\left(Q_{2} \cap X_{1} \cap Y_{2}\right) \cup \\
& \cup\left(P_{2} \cap X_{2} \cap Y_{1}\right) \cup\left(Q_{1} \cap X_{2} \cap Y_{1}\right) \cup\left(X_{1} \cap X_{2} \cap Y_{1} \cap Y_{2}\right) \\
\subset & \left(P_{1} \cap X_{2} \cap Y_{2}\right) \cup\left(P_{1} \cap X_{2} \cap Y_{2}\right) \cup\left(Q_{2} \cap Y_{1} \cap X_{1}\right) \cup\left(Q_{2} \cap Y_{1} \cap X_{1}\right) \cup \\
& \cup\left(P_{2} \cap X_{1} \cap Y_{1}\right) \cup\left(Q_{1} \cap Y_{2} \cap X_{2}\right) \cup\left(V_{1} \cap V_{2}\right) \\
= & \left(P_{1} \cap V_{2}\right) \cup\left(Q_{2} \cap V_{1}\right) \cup\left(P_{2} \cap V_{1}\right) \cup\left(Q_{1} \cap V_{2}\right) \cup\left(V_{1} \cap V_{2}\right)=\emptyset,
\end{aligned}
$$

by (15) and (16). This contradicts the assumption that $C$ is a continuum. Condition (ii) is then proved, and so is 4.2 .

4.3. Let $T$ be a tree which is not an arc. Let $v_{1}, \ldots, v_{k}$ be all the branch-points of $T$, and let $n_{i}$ denote the ramification order of $T$ at $v_{i}$, i.e. $n_{i}$ is the number of components of $T \backslash\left\{v_{i}\right\}(i=1, \ldots, k)$. Denote

$$
m(T)=\left[\left(\sum_{i=1}^{k} n_{i}\right)-(k+1)\right]^{-1} .
$$

Then there exists a simple triod $T^{\prime} \subset T$ such that $m(T) w(T) \leqslant w\left(T^{\prime}\right)$ (see [9], p. 8).

Let us define separately $m(A)=1$ for each arc $A$. Observe that if $T^{\prime} \subset T$ are trees, then $m(T) \leqslant m\left(T^{\prime}\right)$. The next result follows from 4.1 and 4.3 .

4.4. Corollary. If $T$ is a tree, then $m(T) w(T) \leqslant \sigma(T)$.

Remarks. For some trees, the inequality in 4.4 provides a sharp estimation of the span. Indeed, the 4 -od $T$ from 4.2 has $m(T)=(4-2)^{-1}=\frac{1}{2}$ and $\sigma(T)=\frac{1}{2}$ $=m(T) w(T)$. This estimation, however, is not the best one for all trees. We cite an interesting problem that seems to be important here. It is the following unsettled conjecture of Frances O. McDonald: is it true that each tree $T$ which is not an arc contains a simple triod $T^{\prime}$ such that $w(T) \leqslant 2 w\left(T^{\prime}\right)$ ? If the answer were "yes", we would get, by 4.1 , the inequality $w(T) \leqslant 2 \sigma(T)$ for all trees. Up to now, McDonald's conjecture has been proved for $T$ being " $n$-ods" and $n=4,5,6$ (see [9], p. 13). Thus, for all the 5 -ods and 6 -ods, it already provides a better estimation of the span than that given by 4.4 .

4.5. EXAMPLE. For each $\varepsilon>0$, there exists a simple triod $T$ on the plane $R^{2}$ such that $\sigma(T)=1$ and $w\left(T^{\prime}\right)<\varepsilon$ for each simple triod $T^{\prime} \subset T$.

Proof. There exists an atriodic tree-like continuum $X \subset R^{2}$ such that $\sigma(X)>0$ (see [3], pp. 100 and 106). Moreover, $X$ is "triod-like" in the sense that there are finite open covers of $X$ with the mesh arbitrarily small and with the nerve being a simple triod. These covers can be constructed by means of open disks on the plane, so that their nerves are embeddable in the unions of the disks (ibidem, see also [4], p. 76). It follows (compare 5.1 below) that there exists, for $n=1,2, \ldots$, a $(1 / n)$-translation $f_{n}: X \rightarrow R^{2}$ such that $T_{n}=f_{n}(X)$ is a simple triod. Let $\gamma=\frac{1}{2} \varepsilon \sigma(X)$. We have

$$
\lim _{n \rightarrow \infty} \sigma\left(T_{n}\right)=\sigma(X),
$$

by 3.6 , and therefore there is a positive integer $n_{0}$ such that $\frac{1}{2} \sigma(X) \leqslant \sigma\left(T_{n}\right)$ for $n \geqslant n_{0}$. We claim that there also exists an integer $n_{1} \geqslant n_{0}$ such that $w\left(T^{\prime}\right)<\gamma$ for each simple 
triod $T^{\prime} \subset T_{n}$. If it were not so, each $T_{n}$ (where $n \geqslant n_{0}$ ) would contain a simple triod $T_{n}^{\prime}$ with $\gamma \leqslant w\left(T_{n}^{\prime}\right)$. Hence, by 2.4 , there would exist arcs $A_{\text {in }} \subset T_{n}^{\prime} \subset T_{n}$ $(i=0,1,2)$ such that $A_{0 n} \cap A_{1 n} \cap A_{2 n} \neq \varnothing$ and none of the $\operatorname{arcs} A_{0 n}, A_{1 n}, A_{2 n}$ is contained in the $\gamma$-neighbourhood of the union of the other two. All these arcs are contained in a bounded subset of the plane. Without loss of generality, we can assume that the three sequences of the $\operatorname{arcs} A_{\text {in }}(i=0,1,2)$ converge to some three continua $C_{i} \subset X$ (when $n \rightarrow \infty$ ), respectively. Then also $C_{0} \cap C_{1} \cap C_{2} \neq \varnothing$ and none of the continua $C_{0}, C_{1}, C_{2}$ is contained in the union of the other two, which contradicts the fact that $X$ is atriodic (see [10], p. 443). The existence of $n_{1}$ is thus proved.

Let $p \in R^{2} \backslash T_{n_{1}}$ be a point. We define an embedding $h: T_{n_{1}} \rightarrow R^{2}$ by taking $h(x)$ to be the point of the ray $\overrightarrow{p x}\left(x \in T_{n_{1}}\right)$ such that

$$
\operatorname{dist}[p, h(x)]=\operatorname{dist}(p, x) / \sigma\left(T_{n_{1}}\right),
$$

whence $\operatorname{dist}[h(x), h(y)]=\operatorname{dist}(x, y) / \sigma\left(T_{n_{1}}\right)$ for $x, y \in T_{n_{1}}$. The set $T=h\left(T_{n_{1}}\right)$ is a simple triod with the span $\sigma(T)=\sigma\left(T_{n_{1}}\right) / \sigma\left(T_{n_{1}}\right)=1$. If $T^{\prime} \subset T$ is a simple triod, then

$$
w\left(T^{\prime}\right)=w\left[h^{-1}\left(T^{\prime}\right)\right] / \sigma\left(T_{n_{1}}\right)<\gamma / \sigma\left(T_{n_{1}}\right) \leqslant 2 \gamma / \sigma(X)=\varepsilon .
$$

5. The span of certain tree-like continua. The following well-known lemma establishes a relationship between finite open covers, their nerves and $\varepsilon$-translations.

5.1. LEMMA. Let $X$ be a non-empty subset of the Hilbert space $R^{\omega}$. If $\varepsilon>0$ and $C$ is a finite open cover of $X$ such that $\operatorname{mesh}(C)<\varepsilon$, then there exists a polyhedron $P \subset R^{\omega}$ contained in the $\varepsilon$-neighbourhood of $X$ in $R^{\omega}$ and an $\varepsilon$-transtation $x: X \rightarrow R^{\omega}$ such that $P$ is topologically the nerve of $C$ and $x(X) \subset P$. Moreover, if the nerve of $C$ has dimension $n$, then $R^{\omega}$ can be replaced by the Euclidean $(2 n+1)$-space $R^{2 n+1}$ (see [5], pp. 319, 324 and 330).

Let $\Pi$ be a collection of polyhedra. A compact metric space $X$ is called $\Pi$-like provided, for each $\varepsilon>0$, there exists a finite open cover $C$ of $X$ such that mesh $(C)<\varepsilon$ and the nerve of $C$, if non-degenerate, is topologically a polyhedron belonging to $I I$. Our next proposition involves the McDonald coefficient $m(T)$ as defined in 4.3.

5.2. Let $\Pi$ be a finite collection of trees and let $X$ be a $\Pi$-like continuum. Denote

$$
m(\Pi)=\operatorname{Min}\{m(T): T \in \Pi\} \text {. }
$$

Then $m(I) w(X) \leqslant \sigma(X)$.

Proof. We can assume $X$ is non-degenerate; otherwise 5.2 states a trivial fact. Since then $X$ is one-dimensional, it is embeddable in $R^{3}$. Let us also assume that $X \subset R^{3}$ and, moreover, that the space $R^{3}$ is remetrized so that the metric in $R^{3}$ is an extension of the given metric in $X$ (see [2], p. 353). By 5.1, there exist trees $T_{n} \subset R^{3}$ and $\varepsilon_{n}$-translations $f_{n}: X \rightarrow R^{3}$ such that $\varepsilon_{1}, \varepsilon_{2}, \ldots$ converge to zero, $T_{n}$ is topologically a member of $\Pi$ and $f_{n}(X) \subset T_{n}(n=1,2, \ldots)$. Since $X$ is non-degenerate, all but a finite number of the sets $T_{n}^{\prime}=f_{n}(X)$ are non-degenerate. Consequently, they are trees and the inclusions $T_{n}^{\prime} \subset T_{n}$ imply the inequalities

$$
m(\Pi) \leqslant m\left(T_{n}\right) \leqslant m\left(T_{n}^{\prime}\right) \text {. }
$$

It follows from 3.6 and 4.4 that

$$
\begin{aligned}
m(I) w(X) & =m(\Pi) \lim _{n \rightarrow \infty} w\left(T_{n}^{\prime}\right)=\lim _{n \rightarrow \infty} m(M) w\left(T_{n}^{\prime}\right) \\
& \leqslant \lim _{n \rightarrow \infty} m\left(T_{n}^{\prime}\right) w\left(T_{n}^{\prime}\right) \leqslant \lim _{n \rightarrow \infty} \sigma\left(T_{n}^{\prime}\right)=\sigma(X) .
\end{aligned}
$$

A continuum $X$ is called unicoherent provided the common part of any two continua whose union is $X$ is connected. We say that a continuum $X$ is $P$-unicoherent provided there exists a collection $\Pi$ of unicoherent connected polyhedra such that $X$ is $\Pi$-like. If $f$ is a mapping of a metric space $X$, we denote

$$
\Delta_{f}=\left\{\operatorname{dist}\left(x, x^{\prime}\right): f(x)=f\left(x^{\prime}\right), x, x^{\prime} \in X\right\} .
$$

5.3: THEOREM. If $f: X \rightarrow Y$ is a continuous mapping of a $P$-unicoherent continuum $X$ into an arc-like continuum $Y$, then

$$
[0, \sigma(X)] \subset \Delta_{f} .
$$

Proof. Without loss of generality, let us assume that $X$ is a subset of the Hilbert space $R^{\omega}$ and that the metric in $R^{\omega}$ is an extension of the given metric in $X$ (see [2] p. 353). Also, since $Y$ is one-dimensional (or degenerate), we can assume that $Y \subset R^{3}$. We are given a collection $I I$ of unicoherent connected polyhedra such that $X$ is $\Pi$-like. By 5.1 , there exist polyhedra $P_{m} \subset R^{\omega}$ contained in the $\varepsilon_{m}$-neighbourhoods of $X$ in $R^{\omega}$, respectively, and $\varepsilon_{m}$-translations $f_{m}: X \rightarrow R^{\infty}$ such that $\varepsilon_{1}, \varepsilon_{2}, \ldots$ converge to zero, $P_{m}$ is topologically a member of $\Pi$ and $f_{m}(X) \subset P_{m}(m=1,2, \ldots)$. Similarly, there exist arcs $A_{n} \subset R^{3}$ and $\eta_{n}$-translations $g_{n}: Y \rightarrow R^{3}$ such that $\eta_{1}, \eta_{2}, \ldots$ converge to zero and $g_{n}(Y) \subset A_{n}(n=1,2, \ldots)$. Let $\Phi_{n}: R^{\omega} \rightarrow A_{n}(n=1,2, \ldots)$ be a continuous extension of the mapping $g_{n} f: X \rightarrow A_{n}$ (see [6], p. 332).

Since $P_{m}$ is a unicoherent locally connected continuum, the mapping $h=\Phi_{n} \mid P_{m}: P_{m} \rightarrow A_{n}$ fulfills the condition

$$
\left[0, \sigma\left(P_{m}\right)\right] \subset \Delta_{h}
$$

(see [8], p. 207). Given any number $\alpha \in[0, \sigma(X)]$, there exists a number $\alpha_{m} \in\left[0, \sigma\left(P_{m}\right)\right]$ such that

$$
\left|\alpha-\alpha_{m}\right| \leqslant\left|\sigma(X)-\sigma\left(P_{m}\right)\right|,
$$

and thus we also have $\alpha_{m} \in \Delta_{h}$. Consequently, there exist points $\dot{p}_{m n}, p_{m n}^{\prime} \in P_{m}$ with $\operatorname{dist}\left(p_{m n}, p_{m n}^{\prime}\right)=\alpha_{m}$ and $h\left(p_{m n}\right)=h\left(p_{m n}^{\prime}\right)$, whence

and

$$
\left|\alpha-\operatorname{dist}\left(p_{m n}, p_{m n}^{\prime}\right)\right| \leqslant\left|\sigma(X)-\sigma\left(P_{m}\right)\right| \quad(m, n=1,2, \ldots)
$$

$$
\Phi_{n}\left(p_{m n}\right)=\Phi_{n}\left(p_{m n}^{\prime}\right) \quad(m, n=1,2, \ldots) .
$$

Let $n$ be fixed for this part of the proof. Since the polyhedron $P_{m}$ is contained in the $\varepsilon_{m}$-neighbourhood of $X$ in $R^{\omega}$, there exist points $x_{m n}, x_{m n}^{\prime} \in X$ such that

$$
\operatorname{dist}\left(p_{m n}, x_{m n}\right)<\varepsilon_{m}, \quad \operatorname{dist}\left(p_{m n}^{\prime}, x_{m n}^{\prime}\right)<\varepsilon_{m} \quad(m=1,2, \ldots),
$$

2 - Fundamenta Mathematicae $\mathrm{XCVIII/3}$ 
whence

$$
\lim _{m \rightarrow \infty} \operatorname{dist}\left(p_{m n}, x_{m n}\right)=\lim _{m \rightarrow \infty} \operatorname{dist}\left(p_{m n}^{\prime}, x_{m n}^{\prime}\right)=0,
$$

according to the assumption made about the sequence $\varepsilon_{1}, \varepsilon_{2}, \ldots$ By the compactness of $X$, there exists a sequence $m_{1}<m_{2}<\ldots$ of positive integers such that

$$
\lim _{i \rightarrow \infty} x_{m_{i n}}=x_{n}, \quad \lim _{i \rightarrow \infty} x_{m_{i} n}^{\prime}=x_{n}^{\prime},
$$

where $x_{n}, x_{n}^{\prime} \in X$. (Actually, the sequence $m_{1}, m_{2}, \ldots$ may depend on $n$, but this is irrelevant here, $n$ being fixed.) Thus we also have

$$
\lim _{i \rightarrow \infty} p_{m, n}=x_{n}, \quad \lim _{i \rightarrow \infty} p_{m i n}^{\prime}=x_{n}^{\prime},
$$

and therefore

$$
g_{n} f\left(x_{n}\right)=\Phi_{n}\left(x_{n}\right)=\lim _{i \rightarrow \infty} \Phi_{n}\left(p_{m_{i} n}\right)=\lim _{i \rightarrow \infty} \Phi_{n}\left(p_{m_{i}}^{\prime}\right)=\Phi_{n}\left(x_{n}^{\prime}\right)=g_{n} f\left(x_{n}^{\prime}\right),
$$

by (22). Moreover, it follows from 3.1 that

$$
\limsup _{i \rightarrow \infty} \sigma\left(P_{m_{i}}\right) \leqslant \sigma(X),
$$

and 3.2 implies

$$
\sigma(X) \leqslant \liminf _{i \rightarrow \infty} \sigma\left[f_{m_{i}}(X)\right] \leqslant \liminf _{i \rightarrow \infty} \sigma\left(P_{m_{i}}\right),
$$

since $f_{m_{i}}: X \rightarrow R^{\infty}$ is an $\varepsilon_{m_{i}}$-translation and $f_{m_{i}}(X) \subset P_{m_{t}}(i=1,2, \ldots)$. As a result, we obtain

$$
\lim _{i \rightarrow \infty} \sigma\left(P_{m_{i}}\right)=\sigma(X),
$$

and condition (21) implies that

$$
\operatorname{dist}\left(x_{n}, x_{n}^{\prime}\right)=\lim _{i \rightarrow \infty} \operatorname{dist}\left(p_{m_{i} n}, p_{m_{i} n}^{\prime}\right)=\alpha .
$$

Again, $X$ being compact, there exists a sequence $n_{1}<n_{2}<\ldots$ of positive integers such that

$$
\lim _{j \rightarrow \infty} x_{n_{j}}=x, \quad \lim _{j \rightarrow \infty} x_{n_{j}}^{\prime}=x^{\prime}
$$

where $x, x^{\prime} \in X$. Consequently, we get

$$
\operatorname{dist}\left(x, x^{\prime}\right)=\lim _{j \rightarrow \infty} \operatorname{dist}\left(x_{n_{j}}, x_{n_{j}}^{\prime}\right)=\alpha
$$

and $g_{n_{j}} f\left(x_{n_{j}}\right)=g_{n_{j}} f\left(x_{n_{j}}^{\prime}\right)$ for $j=1,2, \ldots$ But the mapping $g_{n_{j}}$ is an $\eta_{n_{j}}$-translation, whence

$$
\operatorname{dist}\left[f\left(x_{n_{j}}\right), f\left(x_{n_{j}}^{\prime}\right)\right]<2 \eta_{n_{j}} \quad(j=1,2, \ldots),
$$

by (10). The latter inequality yields

$$
f(x)=\lim _{j \rightarrow \infty} f\left(x_{n_{j}}\right)=\lim _{j \rightarrow \infty} f\left(x_{n_{j}}^{\prime}\right)=f\left(x^{\prime}\right),
$$

so that $\alpha=\operatorname{dist}\left(x, x^{\prime}\right) \in \Delta_{f}$. Since $\alpha$ was an arbitrary number of the interval $[0, \sigma(X)]$, we have shown that this interval is a subset of $\Delta_{f}$.

5.4. Corollary. If $\Pi$ is a finite collection of trees and $f: X \rightarrow Y$ is a continuous mapping of a $\Pi$-like continuum $X$ into an arc-like continuum $Y$, then

$$
[0, m(I) w(X)] \subset \Delta_{f} .
$$

\section{References}

[1] C. E. Burgess, Collections and sequences of continua in the plane II, Pacific J. Math. 11 (1961), pp. 447-454.

[2] F. Hausdorff, Erweiterung einer Homöomorphie, Fund. Math. 16 (1930), pp. 353-360.

[3] W. T. Ingram, An atriodic tree-like continulum with positive span, Fund. Math. 77 (1972), pp. $99-107$.

4] - An uncountable collection of mutually exclusive planar atriodic tree-like continua with positive span, Fund. Math. 85 (1974), pp. 73-78.

[5] K. Kuratowski, Topology, vol. I, New York-London-Warszawa 1966.

[6] - Topology, vol. II, New York-London-Warszawa 1968.

[7] A. Lelek, Disjoint mappings and the span of spaces, Fund. Math. 55 (1964), pp. 199-214.

[8] - and L. Mohler, Real-valued continuous functions and the span of continua, Colloq. Math. 32 (1975), pp. 207-209.

[9] F. O Mc Donald, The widths of simple trees, University of Houston Dissertation 1974. 10] R. H. Sorgenfrey, Concerning triodic continua, Amer. J. Math. 66 (1944), pp. 439-460.

WAYNE STATE UNIVERSITY 